\title{
Le devenir des enfants atteints de drépanocytose homozygote traités par hydroxyurée à Brazzaville (Congo)
}

\author{
Homozygous Sickle Cell Children Treated with Hydroxyurea in Brazzaville (Congo)
}

\author{
J.R. Mabiala-Babela $\cdot$ E.R. Nika $\cdot$ L.C. Ollandzobo Ikobo $\cdot$ A.N. Ofamalekou Gnakingue $\cdot$ B.P.S. Ngoulou $\cdot$ \\ S.V. Missambou Mandilou
}

Reçu le 5 avril 2019; accepté le 31 octobre 2019

(C) Société de pathologie exotique et Lavoisier SAS 2019

Résumé Il s'agit d'une étude longitudinale réalisée entre novembre 2015 et septembre 2017 au CHU de Brazzaville. L'étude a porté sur une série hospitalière d'enfants atteints de drépanocytose homozygote et présentant des complications sévères qui imposaient le recours à l'hydroxyurée. Les variables étudiées étaient l'observance thérapeutique, l'évolution de l'état nutritionnel, des indications de l'hydroxyurée, des éléments de l'hémogramme et de l'électrophorèse de l'hémoglobine au cours du traitement, les effets secondaires et les signes de toxicité. La comparaison des moyennes des éléments hématologiques avant le traitement et la fin de l'étude a fait appel au test $t$ de Student, avec un seuil de significativité fixé à 0,05 . Sur 1024 enfants suivis pour drépanocytose, 107 présentaient une indication de traitement par hydroxyurée, soit $10,4 \%$. Ces indications étaient des épisodes anémiques récurrents $(\geq 4)$ dans 62 cas, des accidents neurovasculaires dans 19 cas, des hyperalgies récurrentes dans 17 cas, un priapisme permanent dans quatre cas et un syndrome thoracique aigu dans deux cas. L'observance thérapeutique était bonne pour $89,5 \%$ des enfants traités. Une amélioration clinique rapide et durable était notée dans la majorité des enfants, avec $88,2 \%$ pour les crises hyperalgiques, et $88,7 \%$ pour les crises anémiques, deux cas de récidives de crise neurovasculaire, mais aucune pour le priapisme et le syndrome thoracique aigu. Sur le plan biologique, on a constaté une augmentation significative du taux de l'hémoglobine fotale $(1,2$ à $16,2 \% ; p<0,05)$, de l'hémoglobine (7 à $8,3 \mathrm{~g} / \mathrm{dl} ; p<0,05)$, du volume globulaire

\footnotetext{
J.R. Mabiala-Babela $(\bowtie) \cdot$ L.C. Ollandzobo Ikobo

Faculté des sciences de la santé, université Marien-Ngouabi, Brazzaville, Congo

e-mail : jmabialababela@yahoo.fr

J.R. Mabiala-Babela · E.R. Nika · L.C. Ollandzobo Ikobo

A.N. Ofamalekou Gnakingue · B.P.S. Ngoulou

S.V. Missambou Mandilou

Service de pédiatrie nourrisson,

CHU de Brazzaville, Brazzaville, Congo
}

moyen $(80,8$ à $96 \mathrm{fl} ; p<0,05)$, et une baisse significative du nombre de globules blancs (15 633 à $9872 / \mathrm{mm}^{3}$; $p<0,05$ ) et des plaquettes (387 002 à $324400 / \mathrm{mm}^{3}$; $p<0,05$ ). Les effets secondaires observés étaient essentiellement des vomissements et une thrombopénie dans deux cas chacun, ainsi qu'un cas de céphalée et une neutropénie. Les indications de traitement par hydroxyurée chez l'enfant atteint de drépanocytose à Brazzaville sont nombreuses. Celles-ci sont dominées par les épisodes anémiques récurrents, les accidents vasculaires cérébraux et les crises hyperalgiques. L'excellente évolution de ces complications sous hydroxyurée représente une alternative intéressante dans nos pays à ressources limitées.

Mots clés Enfant · Drépanocytose · Hydroxyurée · Indications · Hôpital · Brazzaville $\cdot$ Congo $\cdot$ Afrique intertropicale

Abstract The objective of this study is to reduce the morbidity of children with homozygous sickle cell disease presenting severe forms. We have conducted a longitudinal study between November 2015 and September 2017 at the Brazzaville University Hospital. Children with sickle cell disease requiring treatment with hydroxyurea were included. The variables studied were therapeutic compliance, evolutive profile of nutritional status, indications of hydroxyurea, electrophoresis of hemoglobin, blood count, and toxicity. The statistical test used was Student test with a significance threshold of less than 0.05 . One thousand twenty-four children were monitored for sickle cell disease, 107 of which had received hydroxyurea $(10.4 \%)$. The indications for hydroxyurea were recurrent anemic attacks $(\geq 4)$ in 62 cases $(57.9 \%)$, neurological crises 19 cases $(17.8 \%)$, recurrent hyperalgesic crises in 17 cases (15.9\%), priapism in 4 cases (3.7), and acute thoracic syndrome in 2 cases $(1.9 \%)$. Therapeutic compliance was good in $89.5 \%$ of them. A rapid and lasting clinical improvement was noted in the majority of patients with hyperalgesic 
attacks (88.2\%) and anemic attacks (88.7\%), two recurrences for the cerebrovascular accidents, and an absence of recurrence of priapism and of the acute thoracic syndrome. From the biological point of view, there was a significant increase in fetal hemoglobin (1.2 to $16.2 \% ; P<0.05$ ), hemoglobin (7 to $8.3 \mathrm{~g} / \mathrm{dl} ; P<0.05)$, mean cell volume ( 80.8 to $96 \mathrm{fl} ; P<0.05$ ) and a significant decrease in mean white blood cell count $\left(15,633\right.$ to $\left.9,872 / \mathrm{mm}^{3} ; P<0.05\right)$ and platelets $(387,002$ to $\left.324,400 / \mathrm{mm}^{3} ; P<0.05\right)$. The signs of toxicity observed were mainly vomiting and thrombocytopenia in two cases each, one case with headache and the other with neutropenia. Indications for use of hydroxyurea therapy in children with sickle cell disease in Brazzaville are common. These are dominated by recurrent anemic seizures, strokes, and hyperalgesic seizures. The excellent evolution of these complications under hydroxyurea represents an interesting alternative in our countries with limited resources.

Keywords Child ' Sickle cell disease · Hydroxyurea · Indications $\cdot$ Hospital $\cdot$ Brazzaville $\cdot$ Congo $\cdot$ Sub-Saharan Africa

\section{Introduction}

En Afrique, la drépanocytose homozygote représenterait trois quarts des 300000 naissances mondiales annuelles d'enfants drépanocytaires [31]. Au Congo, environ 1,25\% de la population présente une drépanocytose homozygote et $22,25 \%$ est hétérozygote [24].

Dans sa forme homozygote, la maladie est responsable de complications aiguës et chroniques. Les premières comprennent des crises vaso-occlusives (CVO) de divers organes, des épisodes anémiques et des infections aiguës ; elles sont responsables d'une létalité importante [12,18-20]. La prévention des crises et des infections est un volet essentiel de la prise en charge de la maladie drépanocytaire. Elle comprend notamment une bonne hygiène de vie, une supplémentation en acide folique et en zinc, une bonne et large couverture vaccinale et une antibioprophylaxie antipneumococcique [4].

Malgré ces mesures, l'enfant drépanocytaire n'est pas toujours à l'abri de complications graves, notamment les accidents vasculaires cérébraux (AVC) ischémiques ou CVO neurovasculaires, les syndromes thoraciques aigus (STA), le priapisme et les épisodes anémiques récurrents (plus de trois crises en moins de deux ans) qui imposent le recours à des thérapies de fond [1]. Au nombre de celles-ci, il y a la greffe des cellules souches hématopoiétiques (CSH), le programme transfusionnel et l'hydroxyurée. Les plateaux techniques des hôpitaux, les ruptures fréquentes d'approvisionnement en produits sanguins et les ressources des pays en développement ne permettent ni greffe de $\mathrm{CSH}$, ni pro- gramme transfusionnel de qualité. L'hydroxyurée apparaît alors la seule alternative thérapeutique de fond [11,21]. Il s'agit d'une molécule dont l'efficacité dans la prévention et donc la réduction des récurrences des complications graves chez l'enfant est démontrée [7,9,15,22]. Nous décrivons le profil évolutif des enfants atteints de drépanocytose homozygote suivis dans notre service et traités par hydroxyurée.

\section{Patients et méthodes}

Il s'agit d'une étude longitudinale portant sur des enfants atteints de drépanocytose homozygote suivis au centre hospitalier et universitaire de Brazzaville (CHUB) dans l'unité de suivi des enfants atteints de drépanocytose qui existe depuis plus de 30 ans. L'identification et l'inclusion des patients se faisaient au fur et à mesure de leur passage à l'unité de suivi ou en cas d'hospitalisation. Les enfants présentant à l'identification une contre-indication à l'hydroxyurée (insuffisance médullaire ou rénale, insuffisance hépatique ou hépatopathie chronique, trouble de l'hémostase) n'ont pas été inclus dans l'étude. Pour des besoins de cohérence, un recul de deux ans de traitement par hydroxyurée a été retenu pour chaque enfant entre novembre 2015 et septembre 2017.

Le suivi portait sur l'observance thérapeutique, le profil évolutif de l'état nutritionnel, des indications de l'hydroxyurée et des paramètres hématologiques, et les effets secondaires et signes de toxicité du médicament.

L'approbation du Comité d'éthique pour la recherche en sciences de la santé (CERSSA) du Congo a été requise avant la réalisation du travail. Dans tous les cas, le consentement éclairé des parents ou du tuteur légal constituait un préalable.

Les données étaient recueillies à partir d'une fiche d'enquête préétablie par un étudiant en fin d'études de médecine supervisé par un MCU-PH.

Le traitement des résultats s'est fait sur le logiciel ÉpiInfo $^{\mathrm{TM}}$ version 7.1.4, et la tabulation et la réalisation des graphes sur le logiciel Excel 2010. Les résultats sont présentés en valeur absolue et relative pour les variables qualitatives et par les paramètres de position (moyenne, écarttype, minimum, maximum) pour les variables quantitatives. Les estimations des pourcentages ont été fournies avec un intervalle de confiance à $95 \%$. Le test $t$ de Student était utilisé pour comparer les paramètres hématologiques des enfants avant et après traitement par hydroxyurée, avec un seuil de significativité fixé à 0,05 .

\section{Résultats}

Sur 1024 enfants suivis pour drépanocytose, 107 enfants de 2 à 15 ans atteints de drépanocytose homozygote ont bénéficié d'un traitement par hydroxyurée. Le tableau 1 présente 


\begin{tabular}{|c|c|c|c|c|}
\hline Caractéristiques des enfants & & Enfants DSS suivis & $\begin{array}{l}\text { Enfants DSS nécessitant } \\
\text { une intensification }\end{array}$ & $(\%)$ \\
\hline Nombre & & 1024 & 107 & 10,4 \\
\hline \multirow[t]{2}{*}{ Sexe } & Masculin & 508 & 52 & 10,3 \\
\hline & Féminin & 516 & 55 & 10,6 \\
\hline \multirow[t]{3}{*}{ Tranche d'âge } & $<5$ ans & 187 & 41 & 2,2 \\
\hline & $5-10$ ans & 451 & 43 & 9,5 \\
\hline & $10-15$ ans & 386 & 23 & 5,9 \\
\hline \multicolumn{5}{|l|}{ Statut scolaire } \\
\hline & Scolarisés & & 63 & \\
\hline & Non scolarisés & & 44 & \\
\hline \multicolumn{5}{|l|}{ Niveau socio-économique } \\
\hline & Bas & & 41 & \\
\hline & Moyen & & 53 & \\
\hline & Élevé & & 13 & \\
\hline
\end{tabular}

Tableau 2 Indications de l'hydroxyurée chez les 107 enfants atteints de drépanocytose homozygote / Indication of hydroxyurea in 107 children with homozygous sickle-cell disease

\begin{tabular}{|lll|}
\hline Indications & $\boldsymbol{n}$ & $\mathbf{\%}$ \\
\hline Crises anémiques récurrentes ( $\geq 4$ en 2 ans) & 62 & 57,9 \\
Crises neurologiques & 19 & 17,8 \\
Crises hyperalgiques récurrentes ( $>$ 4 par an) & 17 & 15,9 \\
Priapisme permanent (au moins 1 épisode) & 4 & 3,7 \\
Syndrome thoracique aigu & 2 & 1,9 \\
Autres indications & 3 & 2,8 \\
\hline
\end{tabular}

Autres indications $=$ association diabète-drépanocytose $(1 \mathrm{cas})$, cardiomyopathie dilatée sévère (1 cas), énurésie primaire rebelle au traitement (1 cas)

la répartition des enfants en fonction du sexe, de l'âge, du statut scolaire et du niveau socio-économique de la famille.

Les indications de l'hydroxyurée sont consignées dans le tableau 2.

Parmi les 107 enfants enrôlés, 96 ont pu être suivis ; les autres ayant abandonné le traitement respectivement au début, à deux et trois mois (six cas) ou perdus de vue (cinq cas).

Le profil électrophorétique de l'hémoglobine des enfants avant et après l'hydroxyurée est reporté dans le tableau 3.

Le suivi était trimestriel, clinique et paraclinique selon un protocole préétabli. Toutefois, en cas de manifestations aiguës entre deux contrôles, l'enfant était hospitalisé et l'événement était signalé lors du contrôle suivant.

La posologie d'hydroxyurée était de $20 \mathrm{mg} / \mathrm{kg}$ par jour quotidiens ou donnés sur deux jours.
L'observance du traitement était bonne chez 85 enfants $(88,5 \%)$ et moyenne (oubli de deux à trois doses dans le même mois) chez 11 enfants (11,5\%).

Le profil évolutif des crises hyperalgiques, des crises anémiques et des crises neurovasculaires au cours du traitement par hydroxyurée est représenté sur la figure 1. Concernant les crises neurovasculaires, deux récidives ont été notées chez deux enfants différents. Dans le premier cas, un AVC ischémique frontopariétal gauche est survenu chez un garçon de 6 ans à 21 mois de traitement. Dans le second cas, il s'agissait d'une fillette de neuf ans avec un AVC ischémique de la région sylvienne droite à 15 mois de traitement, le taux d'hémoglobine $\mathrm{F}$ de ces deux enfants était respectivement de 15,6 et $13,1 \%$. Aucune récidive de syndrome thoracique aigu ni de priapisme n'a été notée.

L'analyse de la figure 2 montre une amélioration de l'IMC rapporté à l'âge. Il a été noté une amélioration au cours du suivi, alors qu'aucune modification de la taille n'a été retrouvée. La synthèse des paramètres hématologiques avant et après traitement par hydroxyurée est résumée dans le tableau 4.

Les effets secondaires se résumaient : aux vomissements, survenus aux premiers jours du traitement avec une évolution favorable sous antiémétique (métoclopramide et endocétran) dans deux cas ; à des céphalées d'évolution favorable après deux semaines sous antalgique (paracétamol) dans un cas ; à une thrombopénie dans deux cas (135000 et 127000 plaquettes $/ \mathrm{mm}^{3}$ ) survenue respectivement entre le $11^{\mathrm{e}}$ et le $15^{\mathrm{e}}$ mois et d'évolution favorable ; et à un cas de neutropénie majeure à $1000 / \mathrm{mm}^{3}$ au $15^{\mathrm{e}}$ mois du traitement, avec une évolution favorable après arrêt temporaire de l'hydroxyurée pendant une semaine, sans transfusion. Aucune toxicité rénale ni hépatique n'a été notée. 
Tableau 3 Profil électrophorétique de l'hémoglobine avant et à 24 mois du traitement / Electrophoretic profile of hemoglobin before and after 24 months of treatment

\begin{tabular}{|c|c|c|c|c|c|}
\hline & \multicolumn{3}{|c|}{ Taux d'hémoglobine $S$} & \multicolumn{2}{|c|}{ Taux d'hémoglobine $F$} \\
\hline & $\begin{array}{l}\text { Avant le traitement } \\
(n=107)\end{array}$ & $\begin{array}{l}\text { À } 24 \text { mois } \\
\text { du traitement }(n=96)\end{array}$ & & $\begin{array}{l}\text { Avant le traitement } \\
(n=107)\end{array}$ & $\begin{array}{l}\text { À } 24 \text { mois } \\
\text { du traitement }(n=96)\end{array}$ \\
\hline$<70 \%$ & $3(2,8 \%)$ & $32(33,4 \%)$ & Nul & $81(75,7 \%)$ & $25(26 \%)$ \\
\hline $70-90 \%$ & $15(14 \%)$ & $44(45,8 \%)$ & $1-10 \%$ & $15(14 \%)$ & $40(41,7 \%)$ \\
\hline$>90 \%$ & $89(83,2 \%)$ & $20(20,8 \%)$ & $>10 \%$ & $11(10,3 \%)$ & $31(32,3 \%)$ \\
\hline
\end{tabular}

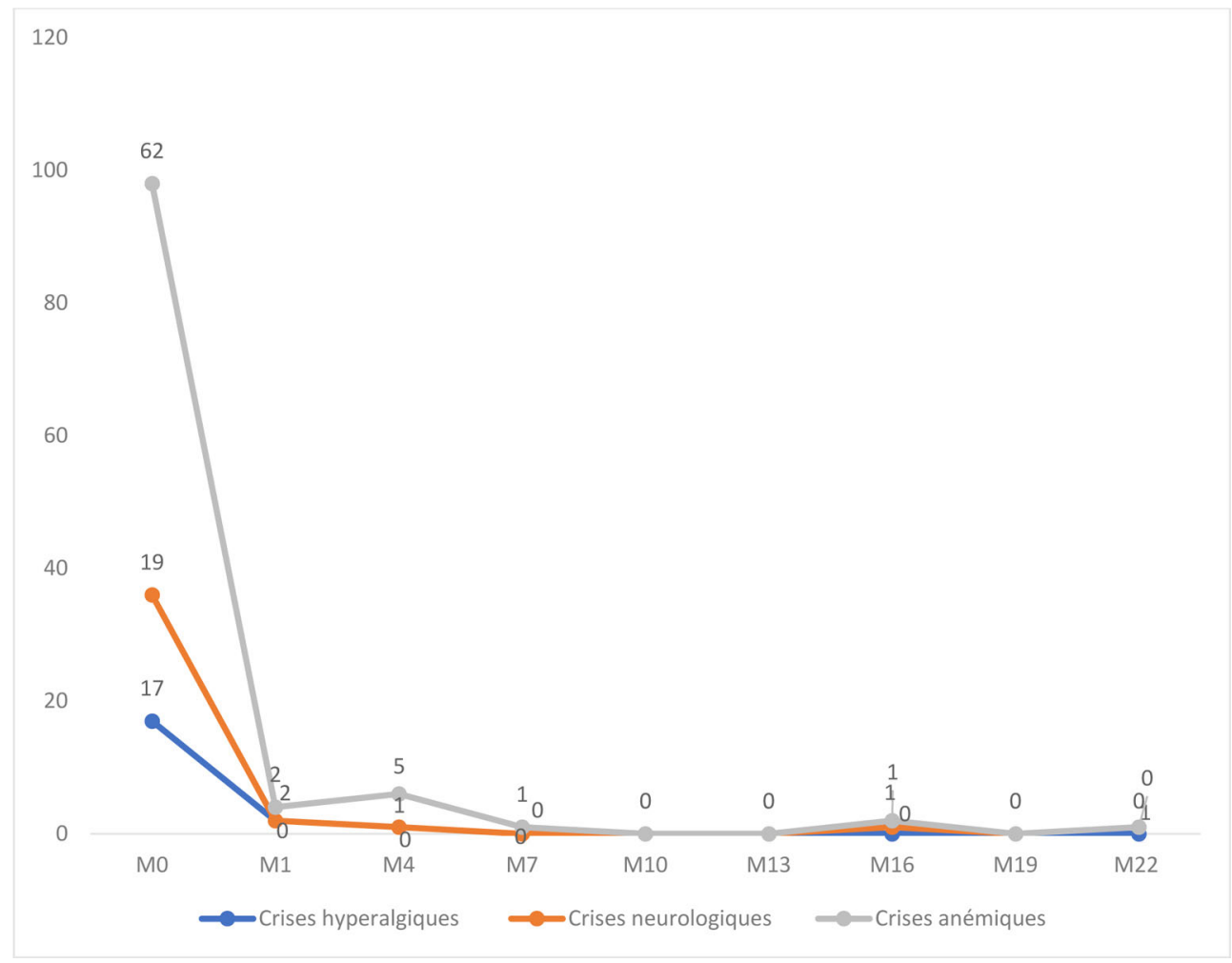

Fig. 1 Profil évolutif des crises hyperalgiques $(n=17)$, neurovasculaires $(n=19)$ et anémiques $(n=62)$ au cours du traitement par hydroxyurée / Evolutive profile of hyperalgesic $(\mathrm{N}=17)$, neurovascular $(\mathrm{N}=19)$ and anemic $(\mathrm{N}=62)$ crisis during the hydroxyurea treatment

\section{Discussion}

Cette étude rapporte les résultats du suivi d'enfants atteints de drépanocytose homozygote présentant des indications de traitement par hydroxyurée conformément aux données de la littérature $[7,15]$. L'étude s'est déroulée au Congo, exclusivement au CHUB de novembre 2015 à septembre 2017. Il aurait été intéressant de pouvoir l'étendre aux autres centres de santé du pays qui prennent en charge des drépanocytaires. Bien que l'étude soit limitée à Brazzaville, elle donne un aperçu sur l'ampleur du problème. En effet, l'unité de suivi des enfants atteints de drépanocytose où a eu lieu l'étude reçoit tous les enfants, voire ceux de l'hinterland, présentant cette pathologie, Brazzaville à elle seule représentant un peu plus du tiers de la population congolaise [23]. La gratuité des contrôles cliniques au sein de cette unité explique que les enfants de toutes les classes socio-économiques soient représentés dans cette série. Les sujets d'étude étant enrôlés au fur et à mesure des consultations de suivi, le recul était variable d'un sujet à un autre, un maximum de deux ans de traitement a été fixé pour cette étude. La posologie prescrite ne pouvait être exacte compte tenu de l'absence de dosages pédiatriques disponibles d'hydroxyurée (présentation dosée à $100 \mathrm{mg}$ ) ; l'absence de laboratoire de pharmacocinétique ne permettait pas non plus de dosage plasmatique du principe actif sur le nycthémère. 


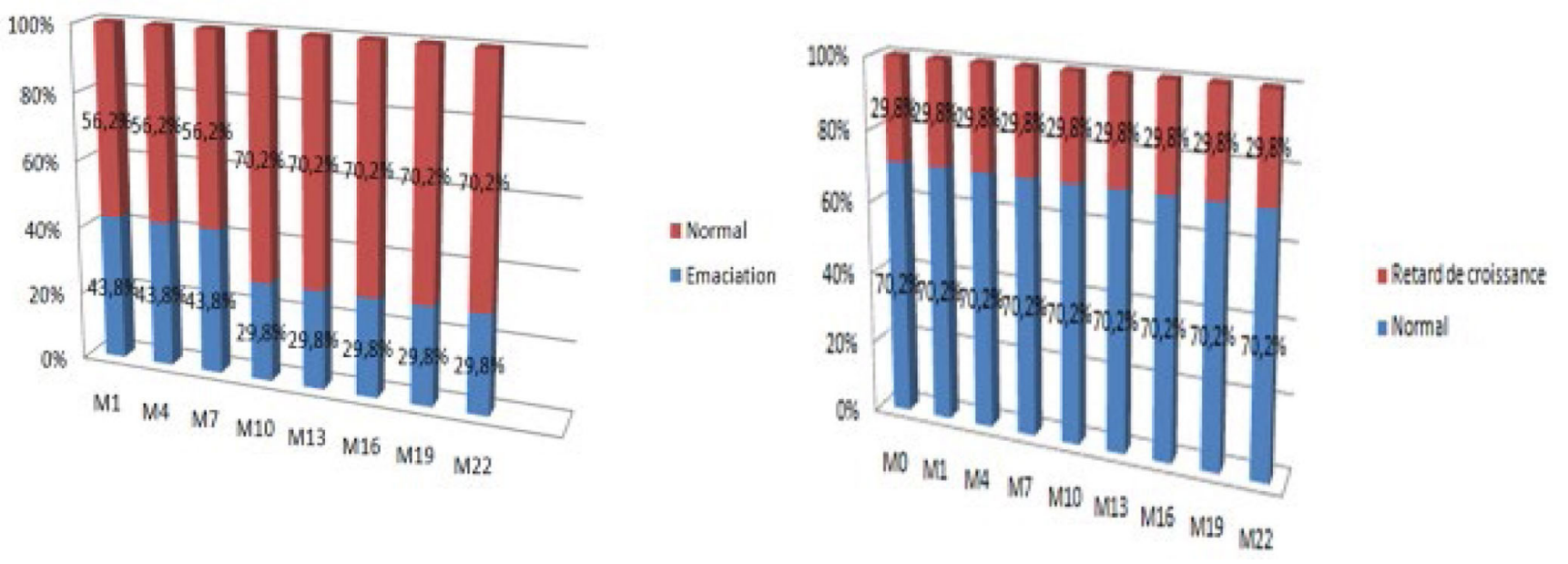

Fig. 2 Évolution de l'état nutritionnel au cours du traitement par hydroxyurée. a): évolution IMC/ÂGE au cours du suivi ; b) évolution T/ÂGE au cours du suivi / Evolution of the nutritional status during the hydroxyurea treatment

Tableau 4 Moyennes des paramètres hématologiques avant et après traitement / Averages of hematologic parameters before and after treatment

\begin{tabular}{|llllll|}
\hline & Hb (g/dl) & Hb F (\%) & VGM (fl) & GB $\left.\mathbf{( m m}^{\mathbf{3}}\right)$ & Plaquettes $\left._{(\mathbf{m m}} \mathbf{3}\right)$ \\
\hline Avant HU & $7,2 \pm 1$ & $1,2 \pm 5,4$ & $82 \pm 6,7$ & $18942,2 \pm 6749,9$ & $387002 \pm$ \\
& & & & & 165685,3 \\
Après HU & $8,3 \pm 1,1$ & $16,5 \pm 4,8$ & $96 \pm 3,7$ & $972 \pm 630,6$ & $324400 \pm 151,6$ \\
Variations & 0,9 & 14,9 & 8,7 & 5761,4 & 62601,8 \\
Valeur de $p$ & $<0,05$ & $<0,05$ & $<0,05$ & $<0,05$ & $<0,05$ \\
\hline
\end{tabular}

Les difficultés de manipulation de la dose maximale tolérée pour les pays en voie de développement ont imposé l'utilisation de la dose minimale $(20 \mathrm{mg} / \mathrm{kg})$ avec rémission clinique, contrairement aux auteurs américains qui vont jusqu'à 35 , voire $40 \mathrm{mg} / \mathrm{kg}[3,15]$. Pour faciliter le traitement, la dose de $20 \mathrm{mg} / \mathrm{kg}$ d'hydroxyurée était donnée un jour sur deux, soit trois à quatre jours par semaine pour les enfants de moins de $20 \mathrm{~kg}$, alors que ceux de plus de $20 \mathrm{~kg}$ bénéficiaient d'une dose de $20 \mathrm{mg} / \mathrm{kg}$ chaque jour. Mellouli et al. en Tunisie dans leur série avaient eu recours au même protocole que nous avec des effets bénéfiques [22]. D'ailleurs, Charache et al. affirmaient que l'effet thérapeutique de l'hydroxyurée n'est pas fonction de la dose maximale tolérée [3].

Au total, 10,4\% d'enfants de la file active suivie au CHUB nécessitaient un traitement par hydroxyurée. L'âge moyen dans cette série était de 7,4 $\pm 3,7$ ans ; extrêmes ( 2 et 15 ans). Le choix des enfants de plus de deux ans était guidé d'une part par la non-disponibilité des formes galéniques pédiatriques, dont l'innocuité n'est pas réellement établie à cet âge $[3,27]$ alors même qu'aucun dosage plasmatique n'était possible. Mellouli et al. [22] avaient fait le même choix en ne débutant le traitement par hydroxyurée qu'au-delà de deux ans. Par contre, des auteurs améri- cains [27] ont utilisé avec succès l'hydroxyurée chez des enfants de moins de deux ans. Dans cette étude, 34,6\% des enfants avaient moins de cinq ans, témoin de la précocité et de la sévérité de la maladie drépanocytaire à Brazzaville comme déjà rapporté dans la sous-région d'Afrique centrale $[18,20]$. Il s'agissait dans 38,6\% des cas d'enfants issus de familles de bas niveau socio-économique et dans 49,5\% de ceux de niveau socio-économique moyen. Cette tendance est conforme aux données nationales selon lesquelles $50 \%$ de la population congolaise vit en deçà du seuil de pauvreté avec un indice de développement humain de 0,592 en 2016 [28]. L'on peut ainsi conclure que l'accessibilité à l'unité de prise en charge des enfants atteints de drépanocytose est bonne puisque le niveau socio-économique des enfants qui y accèdent est le reflet de celui de la population générale.

Les crises hyperalgiques, complication la plus fréquente de la maladie, sont responsables d'un absentéisme scolaire et contribuent à la dégradation de la qualité de vie de l'enfant drépanocytaire [17]. Aussi, la prévention des crises douloureuses hyperalgiques et récurrentes constitue la principale indication de l'hydroxyurée [4]. Pour 17 enfants de cette série, la réduction de celles-ci a été notée chez 15 $(88,2 \%)$ d'entre eux. Ce résultat est superposable à celui 
d'Elira Dokekias et al. [9], de De Montalembert et al. [7] et de Jayabose et al. [15], pour qui, une réduction des crises douloureuses a été retrouvée respectivement chez 62 patients sur 65,27 enfants sur 28 et chez $65 \%$ des patients.

Les crises anémiques sont une complication fréquente chez l'enfant atteint de la drépanocytose à Brazzaville comme partout ailleurs en l'absence de prévention et de traitement. Nous avons obtenu une réduction de crises anémiques chez 55 enfants sur 62 . Ce résultat incite à l'optimisme dans les conditions d'exercice caractérisées au Congo par de fréquentes ruptures en produit sanguin, mais aussi du caractère non déleucocyté, non phénotypé des produits sanguins habituellement utilisés dans nos pays. Bijan et al. [2] ainsi que Jayabose et al. [15] ont, dans leurs études, noté également une diminution significative du taux de transfusion sanguine.

L'AVC est la complication neurovasculaire la mieux documentée et relativement fréquente au cours de la maladie drépanocytaire [4]. Chez 19 enfants de cette étude, seuls deux d'entre eux ont présenté une récidive. Nos résultats corroborent ceux de De Montalembert et al. [8] ainsi que Ware et al. [31], qui respectivement sur 6 et 16 patients, avaient observé une récidive de l'AVC chez deux et trois enfants. Malgré l'efficacité prouvée de l'hydroxyurée dans la prévention secondaire de l'AVC, l'échange transfusionnel et la greffe des CSH, lorsqu'ils sont possibles, demeurent des thérapies optionnelles pour la prévention des récidives $[5,10]$.

La fréquence du priapisme est probablement sous-estimée dans notre étude en raison de l'ignorance de la prise en charge médicochirurgicale de celui-ci par la population à Brazzaville [26]. Il a constitué une indication de l'hydroxyurée avec succès dans quatre cas de cette série. Saad et al. [29] rapportent dans leur étude une absence de récidive du priapisme sous hydroxyurée.

Le syndrome thoracique aigu représente la deuxième indication de mise sous hydroxyurée d'après De Montalembert [4]. Nous y avons recouru avec succès chez deux enfants. Les difficultés diagnostiques en raison de l'absence de définition consensuelle peuvent expliquer la sousestimation de cette entité. En effet, dans une étude hospitalière portant sur les CVO au CHUB, Mabiala-Babela et al. [19] ont colligé 82 (14\%) cas de STA sur 587 épisodes de CVO chez l'enfant et l'adolescent. Alors que d'autres auteurs estiment à près de $40 \%$ la fréquence du STA [25]. Mellouli et al. [22] n'ont pas noté de récidive de STA dans leur étude portant sur sept cas.

Dans notre étude, 43,8\% d'enfants étaient dénutris (rapport poids/taille en deçà de-2ZS) et 29,8\% présentaient un retard de croissance. Ce constat est le même que celui fait par Mabiala-Babela et al. [16] qui rapportaient $97,3 \%$ des patients dont les valeurs anthropométriques se situaient entre le $25^{\mathrm{e}}$ et le $75^{\mathrm{e}}$ percentile. De même, Henderson et al. [14] rapportent $25 \%$ d'enfants atteints de drépanocytose qui pré- sentent des valeurs du poids, de la taille et le rapport poids/ taille inférieur au $5^{\mathrm{e}}$ percentile par rapport aux normes de la NCHS (National Center Health Statistics). Chez les enfants traités par hydroxyurée, aucun effet bénéfique n'a été noté sur le retard statural ; par contre, l'état nutritionnel (rapport poids/taille) est passé progressivement de l'émaciation à la normalisation. Pour Scott et al. [30], le traitement par hydroxyurée avait un bénéfice sur le poids et non pas sur la croissance staturale.

L'amélioration clinique de l'enfant drépanocytaire est tributaire de l'augmentation du taux de l'hémoglobine $\mathrm{F}$ induite par l'hydroxyurée. En effet, une augmentation du taux moyen de l'hémoglobine $\mathrm{F}$ de $1,2 \pm 5,4$ à $16,5 \pm 4,8$ avec une amélioration clinique a été observée chez $84,2 \%$ des enfants de cette étude. Par contre, chez $26 \%$ d'entre eux, l'augmentation du taux de l'hémoglobine $\mathrm{F}$ était nulle malgré une amélioration clinique. Ce constat a été également fait par Mellouli et al. [22]. Dans ce cas, le bénéfice clinique est rattaché à d'autres effets hématologiques de l'hydroxyurée, souvent constants. Il s'agit de l'augmentation du taux de l'hémoglobine et du volume globulaire moyen, la baisse du taux de globules blancs et du taux de plaquettes [13], comme observé dans cette étude (Tableau 4).

Des effets toxiques de l'hydroxyurée ont été décrits [6]. La toxicité cutanée souvent rapportée n'a pas été retrouvée dans cette étude. Seuls, quelques effets mineurs notamment des vomissements, des céphalées, une thrombopénie et une neutropénie mineures ont été observés. De Montalembert et al. [6] ainsi que Jayabose et al. [15], dans leurs études dont la durée moyenne de suivi respective était de 22 et 23 mois, ont montré que l'administration de l'hydroxyurée à court terme n'induit pas de toxicité prononcée. De même, Elira Dokekias et al. [9] ainsi que Ferster et al. [11] dans leurs études couvrant une période de trois ans rapportent une toxicité minime, limitée à une suppression réversible de la moelle. Un recul plus conséquent permettrait de mieux établir l'innocuité de la molécule.

Nous n'avons pas, comme les autres auteurs $[7,15]$, noté de perturbations de la fonction rénale et hépatique.

\section{Conclusion}

Le rythme des complications de la maladie drépanocytaire imposant le recours à l'hydroxyurée est fréquent au Congo. D'où la nécessité d'un suivi rigoureux afin de les identifier. L'excellente évolution de ces complications sous hydroxyurée représente ainsi une indication intéressante dans nos pays à ressources limitées, ce qui impose la mise en place une équipe plus large pour un meilleur suivi de ce traitement.

Liens d'intérêts : Les auteurs déclarent ne pas avoir de liens d'intérêts. 


\section{Références}

1. Aliyu ZY, Tumblin AR, Kato GJ (2006) Current therapy of sickle cell disease. Haematologica 91:7-10

2. Bijan K, Homayon Y, Mohammad B (2016) Hydroxyurea: clinical and hematological effects in patients with sickle cell anemia. Glob J Health Sci 8:252-6. doi: 10.5539/gjhs.v8n3p252

3. Charache S, Dover GJ, Moore RD, et al (1992) Hydroxyurea: effects on hemoglobin $\mathrm{F}$ production in patients with sickle cell anemia. Blood 79:2555-65

4. De Montalembert M (2004) Options thérapeutiques dans la drépanocytose. Rev Prat 54:1557-64

5. De Montalembert M (2007) Échanges érythrocytaires chez les patients drépanocytaires. Hématologie 13:243-9

6. De Montalembert M, Bégué P, Bernaudin F, et al (1999) Preliminary report of a toxicity study of hydroxyurea in sickle cell disease. French Study Group on Sickle Cell Disease. Arch Dis Child 81:437-9

7. De Montalembert M, Belloy M, Bernaudin F, et al (1997) Threeyear follow-up of hydroxyurea treatment in severely ill children with sickle cell disease. The French Study Group on Sickle Cell Disease. J Pediatr Hematol Oncol 19:313-8

8. De Montalembert M, Brousse V, Elie C, et al (2006) Long-term hydroxyurea treatment in children with sickle cell disease: tolerance and clinical outcomes. Hematologica 91:125-8

9. Elira Dokekias A, Okandze Elenga JP, Ndinga J, et al (2005) Évaluation des effets cliniques de l'hydroxyurée chez 132 patients drépanocytaires homozygotes. Tunis Med 83:32-7

10. Estcourt LJ, Fortin MP, Hopewell S, et al (2017) Blood transfusion for preventing primary and secondary stroke in people with sickle cell disease. Cochrane Database Syst Rev 1:CD003146. doi: 10.1002/14651858.CD003146.pub3

11. Ferster BA, Vermylen C, Cornu G, et al (1996) Hydroxyurea for treatment of severe sickle cell anemia: a pediatric clinical trial. Blood 88:1960-4

12. Habibou A, Bachir D, Godeau B (2004) Complications aiguës de la drépanocytose. Rev Prat 54:1548-56

13. Hankins JS, Ware RE, Rogers ZR, et al (2005) Long-term hydroxyurea therapy for infants with sickle cell anemia: the HUSOFT extension study. Blood 106:2269-75

14. Henderson RA, Saavedra JM, Dover GJ (1994) Prevalence of impaired growth in children with homozygous sickle cell anemia. Am J Med Sci 307:405-7

15. Jayabose S, Tugal O, Sandoval C, et al (1996) Clinical and hematologic effects of hydroxyurea in children with sickle cell anemia. J Pediatr 129:559-65

16. Mabiala-Babela JR, Massamba A, Tsiba JB, et al (2005) Composition corporelle d'enfants drépanocytaires homozygotes congolais. Étude longitudinale à Brazzaville, Congo. Bul Soc Pathol Exot 98:394-9 [http://www.pathexo.fr/documents/articles-bull/ T98-5-2825-6p.pdf]

17. Mabiala-Babela JR, Mouyabi Mberi FD, Ollandzobo Ikobo LC, et al (2018) La qualité de vie des adolescents atteints de drépano- cytose homozygote suivis au CHU de Brazzaville (Congo). Bull Soc Path Exot 111:46-50. doi: 10.3166/bspe-2018-0006 [http:// www.pathexo.fr/documents/articles-bull/2018_111_46.pdf]

18. Mabiala-Babela JR, Nkanza-Kaluwako SAT, Ganga-Zandzou PS, et al (2005) Causes d'hospitalisation des enfants drépanocytaires : influence de l'âge (CHU de Brazzaville, Congo). Bull Soc Pathol Exot 98:392-3 [http://www.pathexo.fr/documents/articles-bull/ T98-5-2767-2p.pdf]

19. Mabiala-Babela JR, Nzingoula S, Senga P (2005) Les crises vaso-occlusives drépanocytaires chez l'enfant et l'adolescent à Brazzaville, Congo. Étude rétrospective de 587 cas. Bull Soc Pathol Exot 98:365-70 [http://www.pathexo.fr/documents/articles-bull/T98-5-2696-6p.pdf]

20. Mabiala-Babela JR, Pandzou N, Moyen G (2011) Les manifestations inaugurales de la drépanocytose au CHU de Brazzaville. Ann Afr Med 4:711-7

21. McGann PT, Tshilolo L, Santos B, et al (2016) Hydroxyurea therapy for children with sickle cell anemia in Sub-Saharan Africa: Rationale and Design of the REACH Trial. Pediatr Blood Cancer 63:98-104. doi: 10.1002/pbc.25705. Epub 2015 Aug 14

22. Mellouli F, Bejaoui M (2008) L'utilisation de l'hydroxyurée dans les formes sévères de la drépanocytose : étude de 47 cas pédiatriques tunisiens. Arch Pediatr 15:24-8. doi : 10.1016/j.arcped. 2007.09.013

23. Ministère du Plan, de l'Aménagement du territoire et de l'Intégration (2017) RGPH, CNSEE, République du Congo, Brazzaville

24. Mpemba Loufoua AB, Makoumbou P, Mabiala Babela JR, et al (2010) Dépistage néonatal de la drépanocytose au Congo Brazzaville. Ann Univ M Ngouabi 11:21-5

25. Neonato MG, Guilloud-Bataille M, Beauvais P, et al (2000) Acute clinical events in 299 homozygous sickle cell patients living in France. French Study Group on sickle cell disease. Eur J Hematol 65:155-64

26. Okoko AR, Odzébé ASW, Moyen E, et al (2013) Priapisme chez l'enfant et l'adolescent drépanocytaire homozygote à Brazzaville. J Urol 24:57-61. doi :10.1016/j.purol.2013.04.021

27. Powars DR (2001) Hydroxyurea in very young children with sickle cell anemia is not a cure-all. J Pediatr 139:763-4

28. PNUD (2016) Rapport sur le développement humain 2016. New York, NY 10017, États-Unis, 43 p

29. Saad ST, Lajolo L, Gilli S, et al (2004) Follow-up of sickle cell disease patients with priapism treated by hydroxyurea. Am J Hematol 77:45-9

30. Scott JP, Hillery CA, Brown ER, et al (1996) Hydroxyurea therapy in children severely affected with sickle cell disease. J Pediatr 128:820-8

31. Ware RE, Zimmerman SA, Schultz WH (1999) Hydroxyurea as an alternative to blood transfusions for the prevention of recurrent stroke in children with sickle cell disease. Blood 94:3022-6

32. WHO (2010) Management of birth and hemoglobin disorders: Report of a Joint WHO March of Dimes Meeting, World Health Organization, Geneva, Switzerland 